\title{
Understanding the Paradigm Challenges Posed by Multiplex Panel Testing for Cancer Susceptibility
}

\author{
Mark Robson
}

Published online: 25 September 2014

(C) Springer Science + Business Media New York 2014

\begin{abstract}
Massively parallel ("next generation") sequencing (MPS) is a technology that has revolutionized genetics. In clinical cancer genetics, MPS affords the opportunity to concurrently analyze the sequence of an arbitrary number of genes that may be related to a clinical presentation, such as breast cancer. This multiplex panel testing may identify germ line pathogenic variants in genes that would not have been tested on phenotypic grounds. While this type of testing may be beneficial in specific circumstances, broad application introduces challenges that cannot easily be addressed by conventional pre-test counseling models. Newer approaches are required to preserve the tradition of shared decision-making that has guided clinical cancer genetics since its inception.
\end{abstract}

Keywords Genetic testing - Cancer susceptibility · Multigene testing - Multiplex testing - Panel testing . Genetic counseling

\section{Introduction}

The standard model of genetic counseling for cancer susceptibility testing

\footnotetext{
M. Robson ( $\square)$

Clinical Genetics and Breast Medicine, Memorial Sloan

Kettering Cancer Center, 1275 York Avenue, New York,

NY 10065, USA

e-mail: robsonm@mskcc.org

M. Robson

Weill Cornell Medical College, 1275 York Avenue, New York, NY 10065, USA
}

Genetic testing for cancer predisposition is now a routine part of oncologic care. Only a small fraction of all cancer patients develop their disease as the result of an inherited genetic variant. For these uncommon individuals, however, the identification of the specific allele associated with their cancer can have substantial clinical utility. Clinical utility has various definitions, but in general a test can be said to have clinical utility if knowing the result of that test will improve clinical outcomes. There are a number of domains wherein a test result can have a positive effect. Most directly, the test may indicate sensitivity to a specific treatment that improves outcome or reduces toxicity compared to an empirically selected therapy. In this context, the test result is a traditional biomarker. This has not been the usual role of cancer susceptibility testing, but this potential role has come to the fore with the demonstration of the activity of inhibitors of poly-(ADP-ribose)polymerases (PARP) in patients with BRCA mutationassociated cancers. This has led to the need for germ line genetic testing as a companion diagnostic to identify the patients who may benefit from these agents [1]. A more obvious, and common, use of germ line information in the treatment of cancer patients is in the prediction of the risk of second primaries. While this knowledge is most commonly thought of as influencing decisions about preventive surgeries or enhanced surveillance in cancer survivors, test results may impact primary treatment in newly diagnosed patients (e.g., the choice of whether to have breast conservation or bilateral mastectomy in hereditary breastovarian cancer, or the choice of limited or more extended colectomy in Lynch Syndrome). Finally, of course, family members can use test results to inform pre-symptomatic testing. Unaffected individuals who share the familial predisposition may enter surveillance programs that are considerably more intensive than those followed by 
patients at population risk, or may even choose to undergo preventive surgeries. And family members who do not share the predisposition are thought to be at or near the cancer risks of the general population, unless there are other personal or familial risk factors at play.

It is important to recognize that the evidence supporting different applications of genetic testing varies widely in extent and quality. In some cases, such as in the use of a genetic test as a companion diagnostic for the use of a particular drug, the evidence is rigorous as for other biomarkers in standard use. However, for most applications, the evidence for clinical utility is more limited, and very few studies have demonstrated an improvement in survival resulting from interventions guided by a genetic test for cancer predisposition. For this reason, considerations of personal utility are important in guiding the decision whether or not to undergo genetic testing. Personal utility is a generic descriptor that encompasses the elements of decision-making which are not directed by considerations of medical outcomes. These elements are often poorly understood, even by the person undergoing testing, and the process of pre-test genetic counseling is critical to clarify these aspects and facilitating decision-making about whether or not to accept testing, especially in situations where clinical utility is limited. When cancer susceptibility testing first became available, considerations of personal utility dominated as the understanding of risks was somewhat unsophisticated, and the effectiveness of interventions was not clear. As the evidence base has evolved, the relative importance of clinical and personal utilities as reasons for undergoing testing has also changed, at least for some genes. For example, in a young woman with small, newly diagnosed "triple-negative" breast cancer and a family history of breast and ovarian cancer, the detection of a pathogenic $B R C A l$ sequence variant would have a significant impact on surgical treatment options, and clinical considerations dominate the decision whether or not to undergo genetic testing. For that woman's unaffected 24-year-old sister, however, considerations of personal utility are of paramount importance. The genetic counseling requirements for these two circumstances are profoundly different, but for all genetic testing the principle of respect for persons mandates context-appropriate pre-test education and informed consent [2-4].

\section{The Advent of Multigene Panel ("Multiplex") Testing}

The traditional approach to genetic testing for cancer risk has been iterative, with the clinician deciding what gene is most likely to be altered in a family on the basis of the "phenotype" of that family (usually, the personal and family cancer history). The clinician counsels the proband about the implications of a mutation in that gene, then, with consent, performs testing [2-4]. If the test reveals no causative sequence variant, then the clinician re-evaluates, decides whether to pursue another gene, and, if indicated, begins the process again. The advantage of this approach is that the clinician can constrain testing to those genes that he or she believes will be of benefit in the particular circumstance, and the person (and family) being tested is able to understand and consent to that judgment. A disadvantage is that the approach can be insensitive as the clinician may be mistaken in the selection of genes to test, or the family history may be atypical and not indicative of a particular gene mutation. This approach can also be slow and expensive, if multiple iterations are required to reach a conclusion.

Massively parallel ("next-generation") sequencing (MPS) is a profoundly disruptive technology that facilitates rapid sequencing of a large number of genes. In clinical cancer genetics, MPS can be used to concurrently analyze the sequence of a set of genes chosen to represent the possible causes of a particular presentation of personal or family cancer history. The advantages and disadvantages of this approach are nearly the exact opposites of the traditional iterative approach. The panel-based approach is highly sensitive, and early studies demonstrate that this testing identifies a significant number of sequence variants that likely would not have been discovered with the traditional approach (unfortunately including a significant

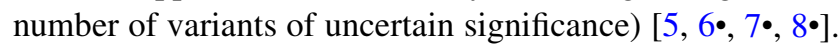
Although there are (as yet) no formal cost-effectiveness analyses, it is possible, even likely, that the cost per identified variant is less than the traditional iterative approach. A major disadvantage of the panel approach relates to the challenges of applying the traditional pre-test consent model to this new technology [9]. When there are many genes on a panel, it is impractical to expect the clinician to be able to communicate (or the patient to absorb) the traditional elements of pre-test consent for each of the genes being tested. Furthermore, neither the clinician nor the person being tested has the ability to select the genes tested in most multiplex panels, which may include a number of genes that have no clear relation to the presenting "phenotype" of the proband and his or her family. As a result, panel-based testing represents a distinct shift away from the historical shared decision-making model of cancer susceptibility testing toward a more directive approach with patient assent, which is closer to the conventional model of medical diagnostic testing (e.g., ordering a CT scan as part of a diagnostic evaluation), which does not generally involve an extensive informed consent process. 


\section{Clinical Utility and Multiplex Panel Testing}

As noted earlier, non-directive pre-test counseling becomes less central as the clinical utility of a genetic test increases. Indeed, at some point, non-directive counseling becomes almost inappropriate if the results of a test are of direct relevance to the selection of treatment. The difficulties of obtaining specific consent for testing of each of the genes on a multiplex panel would be less problematic if each of the individual genes was of clear clinical utility. For instance, hereditary pheochromocytoma-paraganglioma is a genetically heterogeneous syndrome that can result from sequence variants in a number of different genes [10, 11], but associations between particular phenotypes and specific genes are incomplete (apart from VHL and MEN2). Identifying a causative sequence variant in an affected individual may be of limited clinical utility for that person, but greatly increases the informativeness of pre-symptomatic testing for his or her unaffected family members, as those who do not share the variant are at the population risk for this rare disorder. This is a circumstance where panel testing is a logical approach. Another is simultaneous analysis of all mismatch repair genes in individuals suspected of having Lynch Syndrome, when tissue is not available to direct more specific testing through immunohistochemical analysis for loss of the encoded proteins. A third circumstance where panel testing may be appropriate is if the proband's personal or family histories suggest the possible presence of more than one "high penetrance" syndrome. In this situation, concurrent testing for the potentially responsible genes may be more time and costefficient (although specific informed consent for the genes analyzed should still be possible).

Multiplex testing, then, may be advantageous if a syndrome is genetically heterogeneous but phenotypically restricted (and if a pathogenic variant in any causative gene will have the same degree of clinical utility), or if an individual's presentation may plausibly result from more than one syndrome. In both of these situations, it is feasible to prepare the individual being tested for each possible result of the testing, and genes are only tested if they are directly relevant to the individual's circumstance. Most individuals presenting for risk assessment do not clearly fall into either of these categories, however. And most commercially available multiplex panels are not designed to address these particular applications of the technology.

As currently designed, most panels contain three categories of genes. The first group is the so-called high-penetrance genes that could plausibly be related to the personal or family history of the proband. These genes usually cause recognizable autosomal dominant syndromes, and are generally accepted as being of established clinical utility. Variants in these genes are relatively unlikely to be found, however, in the absence of a suggestive family history (although limited family structure or lack of information about family members' diagnoses can obscure the syndromic presentation) [12]. Other genes on many panels are high-penetrance genes that are associated with syndromes other than those suggested by the patient's personal or family history. While sequence variants in these genes are of clear clinical utility when found in the context of a suggestive family history, the implications are less clear when such a history is absent. For example, identifying a pathogenic BRCA2 sequence variant, while screening a colon cancer family is similar to identify one while conducting population screening. There is uncertainty about the penetrance of variants found in this way, although there is good evidence that there are genetic modifiers of $B R C A 2$ risk, and that penetrance varies with family history [13, 14]. Therefore, interventions (such as preventive mastectomy) that are appropriate to consider in the syndromic context may not be as clearly indicated in this context. Another example is the identification of pathogenic or likely pathogenic $\mathrm{CDHl}$ variants, while evaluating a woman with early onset infiltrating ductal carcinoma but without a family history of gastric or lobular breast cancer. When found in a family with hereditary diffuse gastric cancer, such variants warrant consideration of preventive total gastrectomy [15]. However, it is difficult to apply this recommendation in the absence of a family history without further evidence. The uncertainty about the appropriate medical response to the finding of "out of context" sequence variants may contribute to significant anxiety on the part of the person receiving the result. This may be particularly significant when applying multiplex testing to unaffected individuals, presumably because informative affected family members are unavailable for testing. The intent of pre-test genetic counseling is to mitigate informational risk by providing sufficient information to allow an individual to decide whether he or she is ready to accept results such as these. Unfortunately, the more general, directive counseling that often accompanies panel testing may be less effective in achieving this benefit.

The third category of genes on multiplex panels is the so-called "moderate penetrance" genes. For most of these, case-control studies established associations with cancer risk after laboratory observations suggested the genes as candidates. In the discovery case-control ascertainments, the relative cancer risks resulting from inheritance of a protein-truncating mutation in these genes generally ranged from 2 to 4 . Unlike the "high penetrance" genes, pathogenic variants in these genes are not commonly recognizable as causing autosomal dominant predispositions, in part due to the more limited risks, and are therefore not usually identifiable in the course of a traditional clinical cancer genetics evaluation. Several of these genes are associated 
with Fanconi's Anemia in individuals who are homozygotes or compound heterozygotes for pathogenic alleles (and ATM, of course, causes ataxia telangiectasia when both alleles are pathogenic), but heterozygotes are phenotypically normal except for their increased cancer risks, which are usually site specific, and particularly linked to breast cancer.

A number of studies have found an appreciable prevalence of mutations in "moderate penetrance" genes in patients with either breast or ovarian (especially high grade serous) cancer, unselected for family history [16, 17]. Clinical cancer geneticists have been aware of these associations for several years. And yet, before MPS and multiplex panels, routine cancer risk assessment did not include seeking sequence variants in these genes. In part, the lack of testing reflected the technical challenge (and cost) of individually sequencing a significant number of genes that are phenotypically indistinguishable and are each rarely associated with cancer. But there has also been considerable reservation about the clinical utility of testing for variants in these genes. The associated relative risks in less selected cohorts have been insufficient to clearly warrant interventions beyond those that would be indicated on the basis of family history alone, although the risks in the context of a strong family history may be more relevant. For instance, in a recent segregation analysis of families transmitting pathogenic variants in $P A L B 2$, the average breast cancer risk to age 70 was substantially greater in the presence of close relatives with early onset breast cancer than it was in the absence of such a history (58 vs. $33 \%$ ) [18 ]. This observation, which has also been made in the context of specific mutations in CHEK2, suggests that the impact of pathogenic variants in these genes may be modulated by other co-inherited factors, such as other rare or common variants elsewhere in the genome [19]. If this is the case, it would have important implications for the interpretation of pre-symptomatic testing, as individuals who do not carry the pathogenic variant ("true negative") may nonetheless remain at elevated risk due to inheritance of these as yet undefined modulatory factors.

There are a number of other limitations in the current evidence base that constrains the clinical utility of testing for variants in "moderate penetrance" genes. For most, the age-specific penetrance is unclear, which makes it difficult to advise individuals regarding the optimal timing of interventions such as enhanced surveillance or preventive surgery. For example, while "lifetime" risk estimates for some genes may exceed conventional thresholds for MRIbased breast screening, the existing data do not provide guidance regarding when to begin such screening. For women with breast cancer, information about contralateral cancer risk is lacking for most genes. This is a significant limitation, as delineation of this risk is an important clinical application of $B R C A$ testing in women with newly diagnosed breast cancer. An exception is $C H E K 2$, where analysis of large numbers of women with the 1100delC variant demonstrates a significant contralateral risk, albeit not on par with that experienced by women with $B R C A l$ or $B R C A 2$ mutations [20]. Until similar information becomes available for the other genes, it is difficult to advise newly diagnosed women regarding whether or not they should consider contralateral preventive mastectomy if they are found to carry a pathogenic variant in a "moderate penetrance" gene. Similarly, although protein-truncating variants in RAD51C are linked to an increased risk of ovarian cancer [21], the age distribution of the risk is unclear and therefore it is uncertain whether women with such variants should consider premenopausal salpingo-oophorectomy, with the attendant impact of premature menopause on quality-of-life.

\section{Conclusion}

In summary, multiplex testing for cancer susceptibility in certain specific circumstances offers theoretical advantages over the traditional testing paradigm. Panel-based testing is likely to be advantageous if the personal or family history of the individual being tested may result from pathogenic variation in one or more genes that are known to be relevant to the patient's presentation, and the clinical utility of any identified pathogenic variant is clear. These conditions rarely obtain, however. Panels are increasingly offered without regard for the specific clinical circumstance. While pathogenic variants are likely found more often than with the more traditional approach, the optimal application of the results to patient care remains unclear. For most "moderate penetrance" genes, the understanding of overall and age-specific penetrance and the residual risk to "true negative" patients is inadequate to guide patients making decisions about major interventions such as preventive surgery. The impact on important clinical outcomes such as the development of contralateral breast cancer is similarly inadequate to advise patients regarding their treatment choices. Even pathogenic variants in genes of broadly accepted clinical utility become more difficult to interpret when identified outside of the context of a family history suggesting the syndrome usually connected to such variants. In many ways, then, panel-based MPS introduces more questions into cancer risk assessment than it provides answers. And, at the same time, traditional genetic counseling models that have been applied to share decisionmaking with patients about tests of uncertain clinical utility are inadequate to address the complexities of panel-based testing. Now that multiplex panel testing is available commercially, it is unrealistic to expect that it will only be 
offered in a research context. Nonetheless, it is important for those engaged in cancer risk assessment to remain aware of how much work needs to be done to realize the promise of the new technology, which allows providers to seek pathogenic variants in genes that had not been thought relevant enough to test, and in situations where testing had formerly not been thought to be appropriate [22-24]. This technology challenges the counseling paradigm that has guided cancer risk assessment and susceptibility testing for the past 20 years, and the optimal replacement model has not yet been developed. Providers who are engaged with patients during this transition must remain on guard against being driven by a technological imperative, and work to apply the new approach to further the interests of the families seeking their guidance.

Disclosure M Robson declares no conflicts of interest.

Human and Animal Rights and Informed Consent This article does not contain any studies with human or animal subjects performed by any of the authors.

\section{References}

Papers of particular interest, published recently, have been highlighted as:

- Of importance

1. Turner NC, Ashworth A. Biomarkers of PARP inhibitor sensitivity. Breast Cancer Res Treat. 2011;127(1):283-6.

2. American Society of Clinical Oncology. Statement of the American Society of Clinical Oncology: genetic testing for cancer susceptibility. J Clin Oncol. 1996;14(5):1730-6.

3. American Society of Clinical Oncology. Policy statement update: genetic testing for cancer susceptibility. J Clin Oncol. 2003; 21(12):2397-406.

4. Robson ME, Storm CD, Weitzel J, et al. American Society of Clinical Oncology policy statement update: genetic and genomic testing for cancer susceptibility. J Clin Oncol. 2010;28(5): 893-901.

5. Laduca H, Stuenkel AJ, Dolinsky JS, et al. Utilization of multigene panels in hereditary cancer predisposition testing: analysis of more than 2,000 patients. Genet Med. 2014. doi:10.1038/gim. 2014.40.

6. - Selkirk CG, Vogel KJ, Newlin AC, et al. Cancer genetic testing panels for inherited cancer susceptibility: the clinical experience of a large adult genetics practice. Fam Cancer. 2014. doi:10.1007/ s10689-014-9741-4. Early experiences describing the results of multigene/multiplex panel testing.

7. - Cragun D, Radford C, Dolinsky JS, et al. Panel-based testing for inherited colorectal cancer: a descriptive study of clinical testing performed by a US laboratory. Clin Genet. 2014. doi:10. 1111/cge.12359. Early experiences describing the results of multigene/multiplex panel testing.

8. - Kurian A, Hare EE, Mills MA, et al. Clinical evaluation of a multiple-gene sequencing panel for hereditary cancer risk assessment. J Clin Oncol 2014;32(19):2001-2009. Early experiences describing the results of multigene/multiplex panel testing.

9. Fecteau H, Vogel KJ, Hanson K, et al. The evolution of cancer risk assessment in the era of next generation sequencing. J Genet Couns. 2014;23(4):633-9.

10. Maher E. Phaeochromocytoma and paraganglioma: next-generation sequencing and evolving Mendelian syndromes. Clin Med. 2014;14(4):440-4.

11. Shuch B, Ricketts CJ, Metwalli AR, et al. The genetic basis of pheochromocytoma and paraganglioma: implications for management. Urology. 2014;83(6):1232-2335.

12. Weitzel JN, Lagos VI, Cullinane CA, et al. Limited family structure and BRCA gene mutation status in single cases of breast cancer. JAMA. 2007;297(23):2587-95.

13. Gaudet MM, Kirchoff T, Green T, et al. Common genetic variants and modification of penetrance of BRCA2-associated breast cancer. PLoS Genet. 2010;6(10):e1001183.

14. Begg CB, Haile RW, Borg A, et al. Variation of breast cancer risk among BRCA1/2 carriers. JAMA. 2008;299(2):194-201.

15. Dixon M, Seevaratnam R, Wirtzfeld D, et al. A RAND/UCLA appropriateness study of the management of familial gastric cancer. Ann Surg Oncol. 2013;20(2):533-41.

16. Network Cancer Genome Atlas. Comprehensive molecular portraits of human breast tumors. Nature. 2012;490(7418):61-70.

17. Walsh T, Casadei S, Lee MK, et al. Mutations in 12 genes for inherited ovarian, fallopian tube, and peritoneal carcinoma identified by massively parallel sequencing. Proc Natl Acad Sci. 2011;108(44):18032-7.

18. - Antoniou AC, Casadei S, Heikkinen T, et al. Breast-cancer risk in PALB2. N Engl J Med 2014;371(6):496-506. Demonstration of the style of research needed to bring a "moderate penetrance" gene toward clinical utility.

19. Byrnes GB, Southey MC, Hopper JL. Are the so-called low penetrance breast cancer genes ATM, BRIP1, PALB2, and CHEK2, high risk for women with strong family histories? Breast Cancer Res. 2008;10(3):208.

20. Weischer M, Nordestgaard BG, Pharoah $P$, et al. CHEK $2 * 1100$ delC heterozygosity in women with breast cancer associated with early death, breast cancer-specific death, and increased risk of a second breast cancer. J Clin Oncol. 2012; 30(35):4308-16.

21. Meindl A, Hellebrand H, Wiek C, et al. Germline mutations in breast and ovarian cancer pedigrees establish RAD51C as a human cancer susceptibility gene. Nat Genet. 2010;42(5):410-4.

22. Domchek SM, Bradbury A, Garber JE, et al. Multiplex genetic testing for cancer susceptibility: out on the high wire without a net? J Clin Oncol. 2013;31(10):1267-70.

23. Robson M. Multigene panel testing: planning the next generation of research studies in clinical cancer genetics. J Clin Oncol. 2014;32(19):1987-9.

24. Domchek SM, Nathanson KL. Panel testing for inherited susceptibility to breast, ovarian, and colorectal cancer. Genet Med. 2014. doi:10.1038/gim.2014.56. 